\title{
Variabilité du nombre de rangs de l'épi du maïs
}

\author{
M. Bertrand ${ }^{1}$, F. Tardieu ${ }^{2}$ et A. Fleury ${ }^{1}$ \\ 1 INA-PG, chaire d'agronomie, 78850 Thiverval-Grignon; \\ 2 INRA, laboratoire d'agronomie INA-PG, 78850 Thiverval-Grignon, France
}

(reçu le 20 mars 1989, accepté le 15 septembre 1989)

Résumé - Le nombre de rangs de l'épi de maïs a été mis en relation avec des paramètres climatiques et des critères de croissance, dans 3 séries de situations. La première est constituée de 14 parcelles conduites en bonnes conditions dans divers contextes climatiques (série climat), la deuxième comprend, dans 8 situations climatiques, 3 états structuraux types de la couche labourée, la troisième oppose 2 densités de peuplement. II ressort :

- une liaison entre le nombre de rangs de la série climat et la température moyenne de l'air durant une période qui débute au voisinage de la date d'initiation du phytomère portant l'épi, et s'achève au début de la différenciation des ovules;

- le nombre de rangs de cette série n'apparaît relié ni au rayonnement incident durant cette période, ni à des critères caractérisant la croissance en biomasse:

- une réduction du nombre de rangs dans les parcelles à état structural dégradé, qui n'apparaît pas être liée aux variations de croissance. Le nombre de rangs pourrait donc être déterminé par un mécanisme non directement trophique. Ce point est discuté à partir de résultats bibliographiques.

Zea mays L. - épi - nombre de rangs - température - rayonnement - croissance juvénile - structure du sol

Summary - Variability in ear row number in maize. The row number of maize ear was related to climatic parameters, soil parameters and shoot growth criteria in 3 series of situations (Tables I \& II). The first consisted of 14 experiments carried out at several locations between 1982 and 1988, with favourable soil structure, plant density and fertilization. The second consisted of 8 experiments where 2 typical unfavourable structures of the ploughed layer were compared to the favourable structure $(O)$ in each location. The first typical structure $(B)$ consisted of dm-sized compact clods separated by $\mathrm{cm}$-sized voids, and the second $(C)$ was continuously compacted. In the third series, plant density of over 30 plants. $m^{-2}$ was compared to the common density (between 8 and 9 plants. $m^{-2}$ ).

- in experiments with favourable conditions, the row number was variable (Table $N$ ) and significantly correlated with air temperature during a period which began around the time of the ear leaf was initiated, and ended at the initiation of the first spikelets (Fig. 2 \& 3).

- the row number was neither correlated with cumulated global radiation during the same period (Fig. 2), nor with criteria characterizing plant growth (Fig. 4).

- a reduction in row number was observed in plots with unfavourable soil structure (Table Va). This reduction was not correlated with the reduction in plant growth (Fig. 5). Row number might, therefore, be determined by a non-trophic mechanism.

Zea mays L. - ear - row number - temperature - radiation - early growth - ploughed layer structure

\section{INTRODUCTION}

Le nombre de grains produits par unité de surface de sol rend compte de l'essentiel des variations de rendement en grains du maïs (Yamaguchi, 1974; Boiffin et al., 1976). II apparaît donc dans la majorité des cas comme une composante limitante, bien qu'il soit fixé avant la phase d'accumulation rapide dans les grains (Tollenaar
\& Daynard, 1978). Sa modélisation est donc nécessaire à celle du rendement.

Dans le cas des maïs précoces, où l'épi unique est quasiment de règle, le nombre de grains ne dépend que du nombre de rangs et du nombre de grains par rang. Cette dernière composante est généralement présentée comme la plus variable (Derieux et al., 1984), mais le nombre de rangs moyen de peuplements d'un 
même génotype peut, lui aussi, varier, comme l'observent Navarro (1984) dans une petite région agricole, et Hernandez (1987) pour un réseau national d'essais.

Il est rarement fait mention, dans la bibliographie, de réponse du nombre de rangs à des traitements expérimentaux, hors comparaisons variétales, bien que cette variable soit d'une mesure aisée et souvent utilisée pour évaluer un nombre de grains. Ragland et al. (1966) et Hunter (1980) signalent une augmentation du nombre de rangs avec une photopériode fortement rallongée; Tanaka \& Yamaguchi (1972) notent une légère diminution pour des plantes cultivées à haute densité et sans apport d'azote par rapport à d'autres ne subissant qu'une seule de ces deux contraintes; Schreiber et al. (1962) remarquent une augmentation du nombre de rangs à la suite d'apports azotés sur un sol sableux à faible fourniture en azote; Bonhomme et al. (1984) observent une légère augmentation pour des plantes cultivées sous mulch plastique; Gay \& Roger (1982) n'observent pas de modification du nombre de rangs à la suite de défoliations précoces, antérieures à la différenciation mâle. Par ailleurs, Ruget \& Duburcq (1983) signalent que le nombre de rangs augmente du premier au troisième épi d'une même plante, puis décroît pour les épis plus basaux.

Le nombre de rangs est la première composante fixée dans le temps : dès le début de la différenciation florale, l'organisation du bourgeon d'épi en rangées de fleurs est acquise (Ruget \& Duburcq, 1983). Le nombre de rangs peut donc interférer sur la mise en place des composantes suivantes, et la connaissance de son déterminisme est nécessaire à la modélisation du nombre de grains comme au diagnostic sur les composantes du rendement.
Les sources écologiques de la variabilité $d u$ nombre de rangs étant mal identifiées, l'objet de cette étude est d'explorer cette variabilité, en la mettant en relation avec ce qui est le plus habituellement reconnu comme agissant sur la valeur d'une composante du rendement : des critères de croissance en biomasse et des caractéristiques du milieu.

\section{MATÉRIEL ET MÉTHODES}

\section{Structure des données}

Les données ont été collectées sur un ensemble d'essais conduits, pour l'essentiel, avec d'autres objectifs que l'étude du nombre de rangs. Cette étude relève donc davantage du domaine de l'enquête que de celui de l'expérimentation directe.

Une première série de données est constituée de cultures conduites au champ dans des conditions (densité de peuplement, profil cultural, apports minéraux) visant à éviter toute limitation de la croissance en début de cycle. Les peuplements de cette série sont placés dans différents contextes climatiques du fait du lieu, de l'année et de la date de leur implantation. A ces peuplements au champ a été adjoint un lot de plantes cultivées en serre avec la même exigence de bonnes conditions de croissance. La première source de variation à l'intérieur de cette série est donc d'origine climatique, cette série sera, par la suite, intitulée "série climat" et chaque combinaison lieuannée-date appelée "situation".

Les principales caractéristiques des parcelles et substrat sont présentées Tableau I. Les situations et les densités de peuplement réalisées sont présentées Tableau II, ainsi que les valeurs des principaux paramètres climatiques en début de cycle.

Deux autres séries sont constituées à partir de plusieurs peuplements implantés dans une même situation, et différenciés par des traitements expérimentaux. La seconde série fait varier l'état structural de la couche labourée. Trois traitements ont été réalisés

Tableau I. Caractéristiques des parcelles expérimentales et du substrat utilisé en serre.

\begin{tabular}{|c|c|c|c|c|c|}
\hline \multirow{2}{*}{$\begin{array}{l}\text { Code } \\
\text { G1 }\end{array}$} & \multirow{2}{*}{$\begin{array}{c}\begin{array}{c}\text { Latitude } \\
\text { Nord }\end{array} \\
48^{\circ} 48^{\prime}\end{array}$} & \multicolumn{2}{|c|}{ Longitude } & \multirow{2}{*}{$\begin{array}{c}\begin{array}{c}\text { Profondeur } \\
\text { de sol }(\mathrm{cm})\end{array} \\
180\end{array}$} & \multirow{2}{*}{$\begin{array}{c}\begin{array}{c}\text { Teneur en Argile } \\
(\%)\end{array} \\
18\end{array}$} \\
\hline & & $2^{\circ} 00^{\prime}$ & Est & & \\
\hline G2 & $48^{\circ} 48^{\prime}$ & $2^{\circ} 00^{\prime}$ & Est & 200 & 23 \\
\hline G3 & $48^{\circ} 48^{\prime}$ & $2^{\circ} 00^{\prime}$ & Est & 60 & 28 \\
\hline$M$ & $48^{\circ} 52^{\prime}$ & $3^{\circ} 22^{\prime}$ & Est & 110 & 23 \\
\hline$A$ & $50^{\circ} 15^{\prime}$ & $2^{\circ} 58^{\prime}$ & Est & (a) & (a) \\
\hline LJ & $47^{\circ} 29^{\prime}$ & $1^{\circ} 00^{\prime}$ & Ouest & 80 & 15 \\
\hline$S$ & $48^{\circ} 48^{\prime}$ & $2^{\circ} 00^{\prime}$ & Est & (b) & (c) \\
\hline
\end{tabular}

(a) Données non disponibles

(b) Pots de $5 \mathrm{~L}$

(c) Mélange de volumes égaux d'argile expansé et de tourbe 
Tableau II. Dates et densités de semis, traitements présents dans chaque essai et paramètres climatiques en début de cycle.

\begin{tabular}{|c|c|c|c|c|c|c|c|}
\hline \multirow[b]{2}{*}{ Code } & \multicolumn{2}{|c|}{ Situation } & \multicolumn{2}{|c|}{ Semis } & \multicolumn{2}{|c|}{ Valeurs Climatiques } & \multirow{2}{*}{$\begin{array}{c}\text { Traitements } \\
\text { présents }\end{array}$} \\
\hline & Milieu & Année & Date & $\begin{array}{c}\text { Densité } \\
\mathrm{pl} \cdot \mathrm{m}^{-2}\end{array}$ & $\begin{array}{c}T \operatorname{moy}\left({ }^{\circ} \mathrm{C}\right) / \mathrm{m} \\
0-450^{\circ} j\end{array}$ & $\begin{array}{c}\Sigma R g\left(M J \cdot m^{-2}\right) \\
\quad 80-450^{\circ} j\end{array}$ & \\
\hline $2 g$ & G2 (a) & 82 & $10-05$ & 8,7 & 17,4 & 690 & $\mathrm{O}, \mathrm{B}$ \\
\hline $2 m$ & $\mathrm{M}$ & 82 & $11-05$ & 8,7 & $18,2(b)$ & (c) & $\mathrm{O}, \mathrm{B}, \mathrm{C}$ \\
\hline 3 & G1 & 83 & $03-05$ & 8,7 & 15,3 & 873 & $\mathrm{O}, \mathrm{B}, \mathrm{C}$ \\
\hline $4 a$ & G3 & 84 & $20-04$ & 8,0 & 14,6 & 974 & \\
\hline $4 b$ & G1 & 84 & $31-05$ & 8,7 & 17,0 & 760 & $\mathrm{O}, \mathrm{B}, \mathrm{C}$ \\
\hline $5 a$ & G1 & 85 & $23-04$ & 8,7 & 14,8 & 774 & $\mathrm{O}, \mathrm{B}, \mathrm{C}$ \\
\hline $5 b$ & G3 & 85 & $22-05$ & 8,7 & 16,3 & 755 & \\
\hline A & $A$ & 85 & $28-04$ & 8,0 & 14,0 & 874 (d) & 2 densités \\
\hline $6 a$ & G2 & 86 & $01-05$ & 9,3 & 15,9 & 862 & 2 densités \\
\hline $6 b$ & G1 & 86 & $08-05$ & 8,7 & 16,6 & 845 & $\mathrm{O}, \mathrm{B}, \mathrm{C}$ \\
\hline LJ & LJ & 86 & $13-05$ & 11,2 & 17,5 & (c) & \\
\hline 7 & G1 & 87 & $05-05$ & 8,7 & 15,3 & 678 & $\mathrm{O}, \mathrm{C}$ \\
\hline 8 & G2 & 88 & $30-04$ & 8,7 & 15,4 & 762 & $\mathrm{O}, \mathrm{C}$ \\
\hline$S$ & $S$ & 88 & $04-06$ & (e) & 19,1 & 382 & \\
\hline
\end{tabular}

(a) Code de milieu présenté au Tableau 1; (b) Données thermographiques de la station de la Ferté-Gaucher; (c) Données non disponibles; (d) Données de la station de St Quentin (Aisne); (e) Pots maintenus à distance suffisante pour éviter le recouvrement entre feuilles de plantes voisines.

(Tardieu \& Manichon 1987) : O, de structure fragmentaire formée de mottes de calibre inférieur à $5 \mathrm{~cm}$, masses volumiques moyennes de la couche labourée (p): $1,3-1,4 \mathrm{~g} \cdot \mathrm{cm}^{-3} ; \mathrm{B}$, de structure fragmentaire formée de mottes de dimensions décimétriques et de cavités centimétriques, $p: 1,25-1,50 \mathrm{~g} \cdot \mathrm{cm}^{-3} ; C$, de structure continue et compacte, $p: 1,65-1,75 \mathrm{~g} \cdot \mathrm{cm}^{-3}$. Ces traitements ont été implantés dans 8 situations présentées Tableau II. Ces 8 séries seront par la suite intitulées "séries profils", 3 d'entre elles ne comportent que 2 des 3 traitements. Dans chaque cas le traitement $O$ fait également partie de la série climat.

La troisième série de données oppose 2 densités de peuplement : une voisine de la pratique agricole, 8 à $9 \mathrm{pl} \cdot \mathrm{m}^{-2}$, et l'autre, plus élevée, qui induit une compétition plus précoce. Ces 2 traitements ont été implantés à Arras en 1985 (A), avec deux densités de 8 et 45 $\mathrm{pl} \cdot \mathrm{m}^{-2}$, et à Grignon en $1986(6 \mathrm{a})$ avec 9 et $30 \mathrm{pl} \cdot \mathrm{m}^{-2}$; dans les 2 cas l'écartement entre lignes était maintenu à $0,80 \mathrm{~m}$. Ces 2 couples sont appelés "séries densités", chaque traitement à densité normale appartient aussi à la série climat (Tableau II).

Les plans expérimentaux étaient constitués de 2 ou 3 blocs complets, composés de 60 lignes de semis longues de $30 \mathrm{~m}$ et écartées de $0,80 \mathrm{~m}$. Les prélèvements ont été effectués en respectant une bordure d'au moins $3 \mathrm{~m}$ jusqu'à l'extrémité du bloc, ou jusqu'à la situation d'un prélèvement antérieur.

Dans tous les essais on a utilisé le génotype Borée LG1, hybride simple très précoce, corné-denté. La fertilisation minérale au champ était constituée de 100 unités de $\mathrm{P}_{2} \mathrm{O}_{5}$ et $\mathrm{K}_{2} \mathrm{O}$ enfouis au labour et de 140 à 180 unités d'azote apportées au semis. Le désherbage et le contrôle phytosanitaire ont été conduits conformément aux normes locales de culture. En serre les plantes étaient alimentées en goutte à goutte par une solution nutritive conforme aux indications de Lesaint \& Coïc (1983).

\section{Observations}

Les observations ayant été faites à des dates et stades variables suivant les essais, les comparaisons ont été effectuées en mesurant le temps par une somme de températures, afin de situer les dates d'occurrence des stades de développement.

Les températures maximale et minimale du sol, à $10 \mathrm{~cm}$ de profondeur, ont été mesurées avec un pas de temps de $3 \mathrm{j}$ sur les 3 profils structuraux présents à Grignon en 1983.

Pour les autres essais, seules les températures de l'air étaient disponibles. Ces températures, dans le cas des essais au champ ont été mesurées à $2 \mathrm{~m}$ sous abri, à proximité (moins de $500 \mathrm{~m}$ ) des essais, sauf pour la situation $2 \mathrm{~m}$ où l'on a utilisé des relevés thermographiques (précision $0,5^{\circ} \mathrm{C}$ ) de la station météorolique de la Ferté Gaucher, à $15 \mathrm{~km}$ du lieu d'essai, et altitude voisine. Le suivi des températures en serre est effectué à proximité immédiate des plantes.

La température moyenne journalière $T_{j}$ a été calculée avec la relation $T_{j}=1 / 2\left(T_{x}+T_{n}\right)$, où $T_{x}$ et $T_{n}$ sont respectivement les températures maximale et minimale journalières. Les sommes de températures ont été calculées avec un seuil de $6^{\circ} \mathrm{C}$, à partir du semis.

Les rayonnements globaux utilisés dans les calculs proviennent à Grignon de la station de Trappes située à $10 \mathrm{~km}$ du champ d'essais, et à Arras de la station de St Quentin (Aisne) à $50 \mathrm{~km}$ et environ $0,5^{\circ}$ de latitude plus au sud. Pour l'essai en serre les valeurs de rayonnement ont été estimées à partir des données de la station de Trappes, et d'un coefficient moyen de transmission établi par ailleurs avec un pyranomètre. Dans 2 situations ( $L J$ et $2 \mathrm{~m}$ ) il n'y a pas eu de mesures représentatives du point d'essai.

La date de différenciation femelle a été suivie par prélèvements réguliers pour tous les peuplements des situations $2 \mathrm{~g}, 3,4 \mathrm{a}, 5 \mathrm{a}, \mathrm{A}$ et $6 \mathrm{a}$. Cette initiation débute 
par un allongement du bourgeon d'épi, stade $B$ de l'échelle de Gay \& Ménétrier (1978) inspirée de Bonnett (1940), suivi de l'apparition des ébauches des premières paires d'épillets organisées en rangs, stade $C_{1}$ de la même échelle. Ruget \& Duburcq (1983) notent une durée d'environ $4 \mathrm{j}$ entre ces 2 stades. La notation du stade B étant peu sûre, car il s'agit d'évaluer visuellement si le bourgeon est allongé ou non, nous avons préféré noter l'apparition du stade $C_{1}$. De plus, ce stade $\mathrm{C}_{1}$ correspond à l'extériorisation de l'organisation en rangs, et le nombre de rangs peut alors être considéré comme fixé, ce qui n'est pas acquis au stade $B$.

Des mesures de croissance des parties aériennes ont été effectuées sur 80 à 100 plantes pour chaque date et traitement sauf en $L J$, où les échantillons ne totalisaient que 40 plantes par date et traitement. Les échantillons étaient composés de 6 à 9 placettes de prélèvement. Les plantes ont été étuvées $48 \mathrm{~h}$ à $80^{\circ} \mathrm{C}$ ou $24 \mathrm{~h}$ à $105^{\circ} \mathrm{C}$ suivant les situations. En $4 b, 6 b, 7$ et $S$, il n'y a pas eu de suivi durant la phase du cycle qui nous intéresse ici.

Les prélèvements étant faits à des dates différentes suivant les situations, les comparaisons des états de croissance à une date commune, exprimée en somme de températures ont nécessité une évaluation préalable par interpolation entre les valeurs encadrant cette date. Pour cette interpolation on a préféré utiliser un modèle d'évolution exponentielle de la biomasse en fonction du temps, plus adapté en début du cycle qu'un modèle linéaire (Evans, 1972). Des vitesses de croissance relative (RGR : Relative Growth Rate) moyenne entre deux dates, $a$ et $b$, exprimées en somme de températures, STa et STb, ont été calculées par la relation

$$
\mathrm{RGR}_{a-b}=(\log \mathrm{P} b-\log \mathrm{Pa}) /(\mathrm{ST} b-\mathrm{ST} a)
$$

où $\mathrm{Pa}$ et $\mathrm{Pb}$ sont respectivement les poids moyens mesurés aux dates STa et STb.

Les nombres de rangs ont été comptés à la récolte. Les seules valeurs utilisées ici sont celles mesurées à la base de l'épi, dès le premier étage où l'organisation en rangs est acquise. On ne tient donc compte ni des valeurs correspondant aux tout premiers étages de grains, dans le cas fréquent où ils sont mal organisés en rangs, ni des réductions qu'on observe dans environ 1 cas sur 10, en allant vers le sommet de l'épi. Les comptages ont été faits sur au moins 100 plantes par peuplement. En raison du caractère discret de la variable nombre de rangs, les différences entre distributions ont été évaluées par un test du $\chi^{2}$.

\section{RÉSULTATS}

Le Tableau III présente les dates de différenciation femelle observées dans 6 situations. II apparaît une durée moyenne de $370^{\circ} \mathrm{C} \cdot \mathrm{j}$ avec, autour de cette valeur, des fluctuations atteignant $30{ }^{\circ} \mathrm{C} \cdot \mathrm{j}$, supérieures à la précision des mesures, qui est d'environ $15^{\circ} \mathrm{C} \cdot \mathrm{j}$. Dans une même situation, pour les peuplements soumis à des traitements différents, il n'apparaît pas de retard supérieur à la précision des mesures pour l'initiation femelle des plantes soumises à des conditions défavorables (deuxième et troisième série de situations).
Tableau III. Date de début de différenciation florale.

\begin{tabular}{|c|c|c|c|}
\hline \multirow[t]{2}{*}{ Code } & \multirow[t]{2}{*}{$\begin{array}{c}\text { Date } \\
\text { de semis }\end{array}$} & \multicolumn{2}{|c|}{$\begin{array}{l}\text { Date de début } \\
\text { de différenciation }\end{array}$} \\
\hline & & Calendaire & $\Sigma$ de températures \\
\hline $4 a$ & 20 Avril & 22 Juin & 400 \\
\hline $5 a$ & 23 Avril & 17 Juin & 370 \\
\hline$A$ & 28 Avril & 21 Juin & 350 \\
\hline $6 a$ & 01 Mai & 18 Juin & 380 \\
\hline 3 & $03 \mathrm{Mai}$ & 20 Juin & 340 \\
\hline $2 g$ & 10 Mai & 12 Juin & 365 \\
\hline
\end{tabular}

Les températures du sol mesurées sur les 3 profils en 1983 ne se sont jamais significativement différenciées.

Les valeurs moyennes de nombre de rangs mesurées sur les peuplements de la série climat sont présentées au Tableau IV; celles des peuplements des séries profils et densités respectivement aux Tableaux Va et Vb. II apparaît des différences significatives parmi les peuplements de la série climat. L'effet des traitements sur le nombre de rangs est variable suivant les situations : faible effet de la densité dans un cas, nul dans un autre; diminution moyenne de 0,7 rang pour $\mathrm{B}$ par rapport à $\mathrm{O}$, mais variant de 0,2 (non significatif) à 1,2; diminution moyenne de 0,95 rang pour $\mathrm{C}$ par rapport à $\mathrm{O}$, mais variant de 0 à 1,7 .

La relation entre les fréquences des valeurs individuelles de nombre de rangs et la valeur moyenne, pour tous les peuplements, est présentée à la Figure 1. On note une étroite liaison entre la moyenne et la distribution des valeurs individuelles. Les variations de la moyenne proviennent donc d'une translation de la distribution qui ne se déforme que du fait du caractère dis-

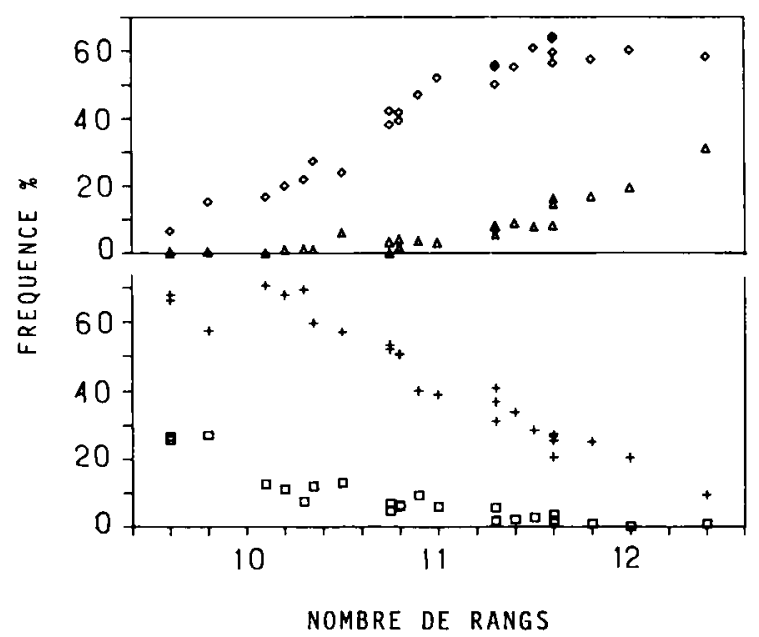

Fig. 1. Relation entre les fréquences d'épis à $8(\square), 10$ (+), $12(\diamond)$ et 14 rangs $(\Delta)$ et la valeur moyenne de nombre de rangs. 
Tableau IV. Nombres moyens de rangs (NR) des peuplements de la série climat

\begin{tabular}{llllllllllllllll}
\hline Code & $2 g$ & $2 m$ & $S$ & 3 & $L J$ & $5 b$ & $4 a$ & $4 b$ & $6 b$ & $5 a$ & $A$ & 7 & $6 a$ & 8 & \\
\hline$N R$ & 12,4 & 12,0 & 11,8 & 11,6 & 11,6 & 11,4 & 11,3 & 11,3 & 11,3 & 11,0 & 10,8 & 10,8 & 10,8 & 10,7 \\
$\left(^{\star}\right)$ & a & ab & bc & bc & $c d$ & $c d$ & $c d$ & $c d$ & $c d$ & de & e & e & e & $e$ & $e$ \\
\hline
\end{tabular}

(") Les nombres suivis de lettres différentes sont significativement différents dans un test $\chi^{2}$ au niveau $5 \%$

Tableau Va. Nombres moyens de rangs des peuplements des séries profils.

\begin{tabular}{|c|c|c|c|c|c|c|c|c|c|c|c|c|c|c|c|}
\hline Code & $2 g$ & $2 m$ & & 3 & & $4 b$ & & $5 a$ & & $6 b$ & & 7 & & 8 & \\
\hline 0 & $12,4 \quad a^{*}$ & 12,0 & $a$ & 11,6 & $a$ & 11,3 & $a$ & 11,0 & $a$ & 11,3 & $a$ & 10,8 & $a$ & 10,7 & a \\
\hline$B$ & $11,6 \quad b$ & 11,6 & $a b$ & 10,9 & $b$ & 10,3 & $b$ & 10,8 & $a b$ & 10,1 & $b$ & & & & \\
\hline$C$ & & 11,5 & $b$ & 10,4 & $c$ & 9,6 & c & 10,5 & $b$ & 9,6 & c & 9,8 & $b$ & 10,7 & a \\
\hline
\end{tabular}

- Les nombres suivis de lettres différentes sont significativement différents dans un test $\chi^{2}$ au niveau $5 \%$.

Tableau Vb. Nombres moyens de rangs des peuplements des séries densités d'implantation.

$\begin{array}{lll}\text { Code } & A & 6 a\end{array}$

$\begin{array}{lrlrl}\text { Densité faible } & 10,8 & \mathrm{a}^{*} & 10,8 & \mathrm{a} \\ \text { Densité forte } & 10,8 & \mathrm{a} & 10,2 & \mathrm{~b}\end{array}$

- Les nombres suivis de lettres différentes sont significativement différents dans un test $\chi^{2}$ au niveau $5 \%$.

cret de la variable nombre de rangs. Par ailleurs les nombres de rangs individuels varient entre les bornes 8 et 14 : on n'observe pas d'épis à 6 ou à 16 rangs. Mais, si l'on assimile la distribution à une distribution gaussienne, on obtient aux bornes de la gamme de variation de la moyenne une fréquence théorique d'apparition d'épis à 6 ou 16 rangs, de l'ordre de $3 \%$, ce qui peut expliquer leur absence dans les échantillons.

Les nombres de rangs moyens par situation ont été mis en relation avec la température moyenne de l'air et le rayonnement global incident, durant plusieurs périodes successives du début du cycle. Du fait d'une mesure du Temps en somme de températures, la température moyenne et le rayonnement incident d'une période sont négativement corrélées : une température élevée réduit la durée calendaire de la période, donc le rayonnement incident. Pour éliminer cet artefact, seuls les coefficients de corrélation partiels entre le nombre de rangs et chacun de ces 2 paramètres ont été utilisés. La Figure 2 présente l'évolution de ces 2 coefficients pour des périodes successives de $50^{\circ} \mathrm{C} \cdot \mathrm{j}$ à partir de la levée. II apparaît une nette liaison entre le nombre de rangs et la température des périodes comprises entre 200 et $350^{\circ} \mathrm{C} \cdot \mathrm{j}$ ( $r:$ de 0,59 à $0,70)$. A l'inverse les coefficients calculés avec le rayonnement ont des valeurs faibles $(r$ de $-0,26$ à $+0,34$ ) et une évolution apparemment erratique.

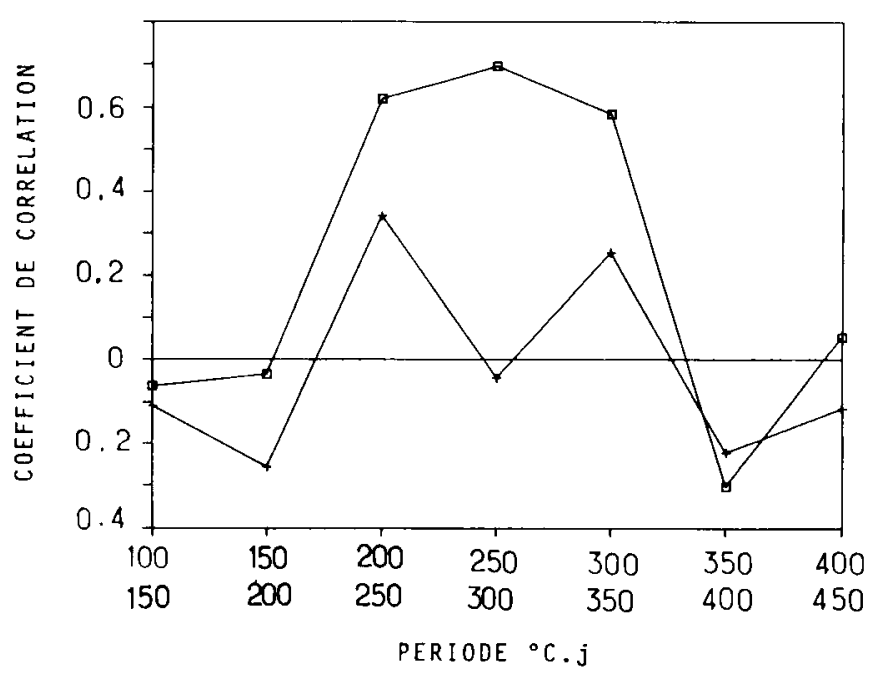

Fig. 2. Coefficients de corrélation partiels entre le nombre moyen de rangs et la température moyenne de l'air ( $\square$ ) ou le rayonnement global cumulé $(+)$ pour differentes périodes exprimées en somme de températures. 
La Figure 3 présente la relation entre le nombre de rangs et la température moyenne pendant cette période de 200 à $350{ }^{\circ} \mathrm{C} \cdot \mathrm{j}$. On observe une bonne liaison $(r=0,85$, HS, en corrélation totale, $r=0,82, \mathrm{HS}$, en corrélation partielle) alors que pour cette même période le nombre de rangs n'est pas corrélé au rayonnement incident $(r=0,44$, NS, en corrélation totale, $r=0,17$, NS, en corrélation partielle). Le choix de périodes de durées ou de positions voisines, à l'intérieur d'une plage allant de 175 à $375^{\circ} \mathrm{C} \cdot \mathrm{j}$, donne des résultats similaires. Dans tous les cas, la situation $5 b$ paraît sortir de la relation, de même qu'à moindre titre la situation $2 \mathrm{~m}$. Dans le cas de $5 \mathrm{~b}$, on ne connait pas de raisons autorisant à penser qu'on a surestimé le nombre de rangs ou sousestimé la température. Dans le cas de $2 \mathrm{~m}$, le fait que les températures utilisées n'ont pas été mesurées sur place peut être à l'origine de cet écart.

Le nombre de rangs a été mis en relation avec la croissance en biomasse aérienne des peuplements durant cette phase où l'on constate une corrélation avec la température, qui suggère une phase de détermination du nombre de rangs. La Figure 4 présente la relation entre le nombre de rangs et la biomasse aérienne par plante atteinte à $350^{\circ} \mathrm{C} \cdot \mathrm{j}$, pour toutes les situations de la série climat où le suivi de croissance permet d'estimer ce poids. II n'apparaît pas de liaison entre ces 2 variables $(r=0,22$, NS), de même qu'entre le nombre de rangs et la vitesse de croissance relative $(r=0,37, \mathrm{NS})$ pour les 8 situations où le suivi de croissance a débuté assez tôt pour permettre le calcul de cette vitesse. La Figure 5 présente la relation entre, d'une part l'intensité d'effet des traitements profils culturaux et densités de semis sur la croissance, caractérisée par l'écart relatif de biomasse à $350{ }^{\circ} \mathrm{C} \cdot \mathrm{j}$, et d'autre part l'écart relatif de nombre de rangs. Là encore, il n'apparaît pas de liaison entre ces deux critères

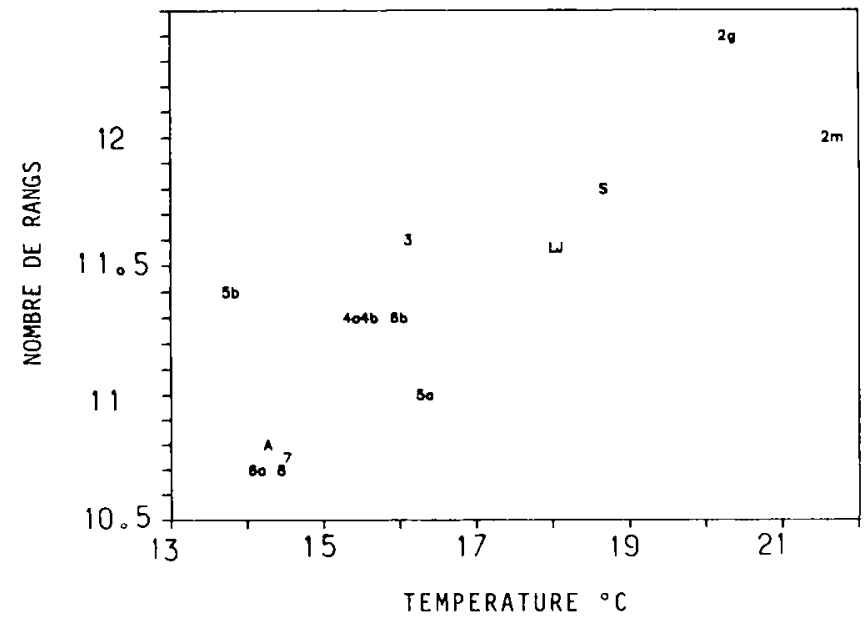

Fig. 3. Relation entre le nombre moyen de rangs des peuplements de la série climat et la température moyenne de l'air entre 200 et $350^{\circ} \mathrm{C} \cdot \mathrm{j}$ après semis.

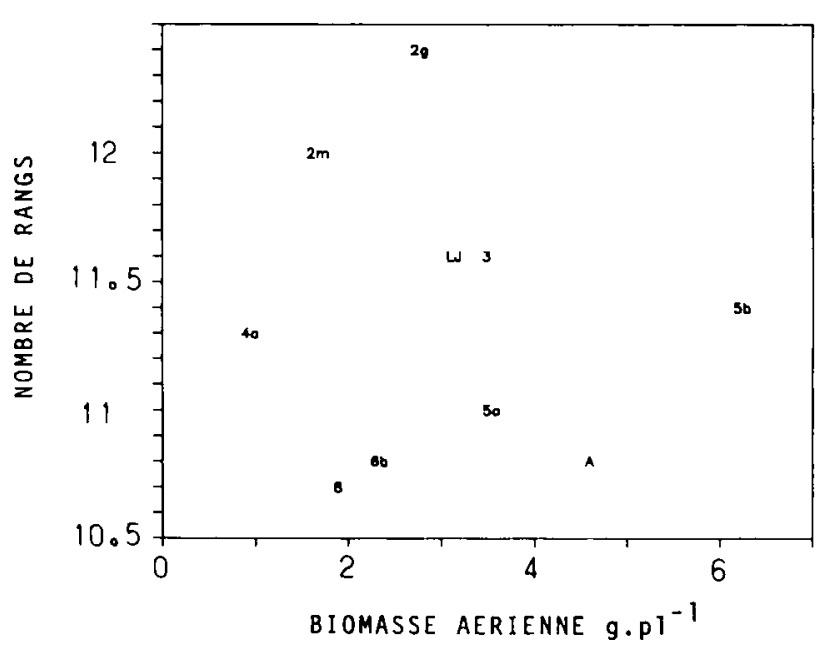

Fig. 4. Relation entre le nombre moyen de rangs des peuplements de la série climat et la biomasse aérienne moyenne à $350^{\circ} \mathrm{C} \cdot \mathrm{j}$ après semis $\left(\mathrm{g} \cdot \mathrm{pl}^{-1}\right)$.

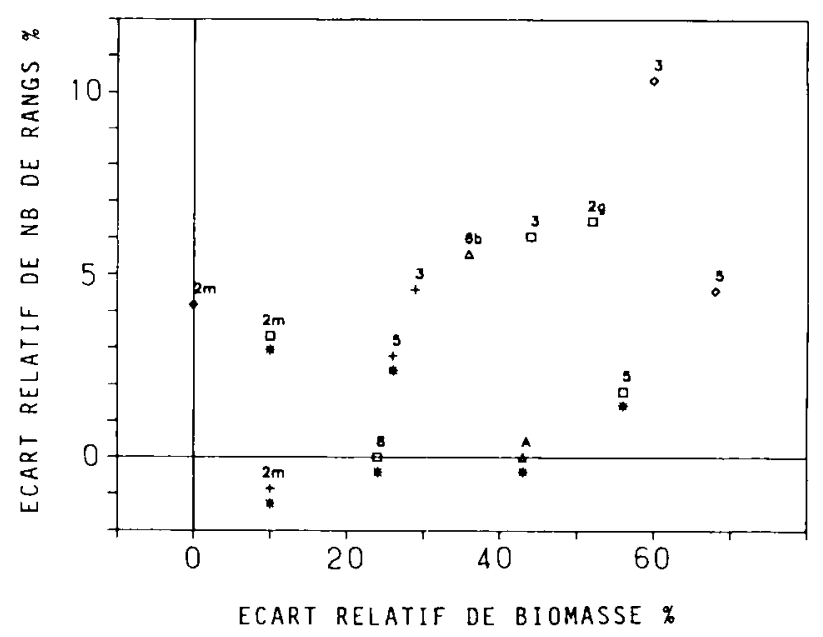

Fig. 5. Relation entre l'écart relatif de nombre de rangs (\%) et l'écart relatif de biomasse aérienne à $350{ }^{\circ} \mathrm{C}$. $j$ après semis $(\%)$ pour les traitements densités $(\Delta), O$ et $B(D), B$ et $C(+)$, $O$ et $C(\theta)$.

* : Ecart de nombres de rangs non significatif.

( $r=0,52 \mathrm{NS}$ ), bien que sur certains traitements la croissance et le nombre de rangs diminuent conjointement.

\section{DISCUSSION ET CONCLUSION}

Les nombres de rangs apparaissent donc reliés à la température qui règne pendant une phase déterminée du cycle. Cette relation intègre des situations provenant de milieux divers, en particulier elle rend compte des valeurs obtenues en serre comme au champ.

La période de réponse du nombre de rangs à la température s'achève à une date compatible avec le début de la différenciation florale, en accord avec les observations de bourgeons qui montrent déjà une organisation en rangs à cette date. Le début de cette période est plus délicat à identifier à un stade de développement, car il est 
assurément antérieur à la différenciation mâle qui intervient pour cette variété aux environs de $300{ }^{\circ} \mathrm{C} . \mathrm{j}$. Cette date intervient donc durant la phase de mise en place des phytomères végétatifs, et en particulier de celui portant le bourgeon d'épi. La date d'initiation de ce bourgeon est difficile à évaluer car on dispose de peu de résultats bibliographiques sur le rythme de fabrication des phytomères.

Pour estimer cette date, on a posé que :

- le nombre de phytomères fabriqués est une fonction exponentielle du temps entre le semis et la différenciation mâle, conformément aux observations de Abbe \& Phinney (1951);

- le nombre de feuilles préformées dans l'embryon est de 5 , valeur généralement observée (Juguet et al., 1986).

Le nombre total de feuilles ayant été en moyenne de 13,5 et le rang moyen de la feuille de l'épi de 8,5 , le calcul mène à une date moyenne d'initiation du bourgeon d'épi voisine de $160{ }^{\circ} \mathrm{C} . \mathrm{j}$. Etant donné ces approximations et hypothèses, la date de début de réaction du nombre de rangs de l'épi à la température peut donc être considérée comme compatible avec la date d'initiation de son bourgeon. Elle intègre la phase de sensibilité du nombre total de feuilles à la photopériode et à la température, peu avant l'initiation mâle (Tollenaar \& Hunter, 1983).

La relation entre le nombre de rangs et la température moyenne reste imparfaite, mais en utilisant une somme de températures pour évaluer la date d'occurrence d'un stade, on doit accepter une certaine imprécision, qui apparaît par exemple pour les dates de différenciation femelle, et qui ne semble pas expliquée par la date de semis. Cette imprécision risque d'être d'autant plus importante ici, que l'on a dû utiliser la température de l'air, moins bonne prédicatrice d'une durée de phase juvénile que celle du sol (Duburcq et al., 1983), et que l'on est, dans certains cas, dans un domaine de température fraîche où la durée d'une phase est mieux estimée par une sommation des $Q_{10}$. De même la mise en relation avec une température plus proche de celle du bourgeon que la température de l'air pourrait donner de meilleurs résultats.

Cette corrélation positive entre le nombre de rangs et la température corrobore donc les observations de Bonhomme et al. (1984), qui notent une légère augmentation du nombre de rangs pour des plantes cultivées sous un mulch plastique qui, d'après Duburcq et al. (1983), induit une élévation de la température de $2{ }^{\circ} \mathrm{C}$, en moyenne.

Cette liaison n'est toutefois pas en mesure d'expliquer les variations de nombre de rangs que l'on constate, dans tous les cas, entre les plantes d'un même peuplement. La pente de la droite de régression, calculée à partir des valeurs présentées Figure 3 , est d'environ $5,8^{\circ} \mathrm{C}$ par rang. La variabilité spatiale et temporelle des températures ne peut donc expliquer des variations pouvant atteindre 6 rangs entre plantes.

La température du sol ne rend pas compte des différences de nombre de rangs constatées entre les 3 types d'états structuraux en 1983. II en est vraisemblablement de même dans les autres situations ou seuls des écarts atteignant 7 à $10^{\circ} \mathrm{C}$ pourraient rendre compte des écarts de nombre de rangs.

Le nombre de rangs n'apparaît lié ni aux différents critères utilisés pour caractériser la croissance en biomasse des plantes, ni au rayonnement, pendant la phase de sensibilité indiquée par la température. Ceci pourrait être lié à une caractérisation trop imprécise de la croissance, en particulier au manque de précision dans la mesure du temps, d'autant plus dommageable que la biomasse suit une évolution exponentielle. De même, on n'a pas pu accéder aux rayonnements interceptés par le peuplement, mais seulement aux rayonnements globaux. D'autre part, pour que la croissance en biomasse de l'ensemble des parties aériennes ait une incidence sur le développement d'un organe, il est nécessaire qu'existe une règle allométrique de répartition des assimilats entre les différents organes exempte de dominance absolue, ce qui n'est pas, a priori, acquis ici. II faudrait alors chercher la valeur prédicatrice de la croissance du bourgeon sur son propre nombre de rangs.

L'autre possibilité est que le nombre de rangs ne soit pas déterminé par un mécanisme seulement trophique, comme le suggère l'absence d'effet de défoliations précoces (Gay \& Roger, 1982). Ce point pose la question du statut anatomique du rang, statut qui peut être différent de celui d'autres composantes du rendement qui sont des ramifications, dont le nombre se fixe par avortement (comme le nombre d'épis du blé par exemple) ou par arrêt d'allongement d'un axe.

Weatherwax (1955) note ainsi que la structure de l'épi ne correspond pas à celle d'une panicule ayant subi une fusion de ses ramifications ("teratological fusion"), mais que les fleurs sont disposées sur des hélices. Cette affirmation est corroborée par les observations de Laubengayer (1949) sur la vascularisation de l'épi, qui ne montrent pas de pôles vasculaires indépendants pour chaque paire de rangs, comme ce devrait être le cas pour une panicule. Cette hypothèse de structure hélicoïdale implique que le nombre de rangs ne serait fonction que du diamètre du bourgeon et de la taille des ébauches de paires d'épillets 
du premier étage, définissant le nombre d'épillets initiés par tour de l'hélice. Or la croissance en épaisseur d'un bourgeon axillaire, du fait des divisions cellulaires, est fortement dépendante de l'activité de régulateurs de croissance, en particulier des cytokinines (Heller, 1978). Ces dernières sont produites par les racines, et d'une manière générale, la balance hormonale peut être modifiée quand le système racinaire est soumis à de fortes pressions liées à l'environnement physique (Lachno et al. 1982), ou quand il colonise des zones à bas potentiel hydrique (Blackman \& Davies, 1985) comme c'est le cas dans les traitements état structural comportant des zones compactées (Tardieu, 1987). De même la température active la production de régulateurs de croissance (Skene \& Kerridge, 1967). En l'absence de liaison claire entre le nombre de rangs et la croissance ou le rayonnement incident, on peut donc supposer que son déterminisme soit en tout ou partie sous contrôle hormonal.

\section{REMERCIEMENTS}

Les auteurs remercient l'ITCF (J.-P. Gillet) et la Société Limagrain (T. Ronsin) pour les résultats obtenus à $L a$ Jaillère et à Arras, ainsi que les étudiants de l'INA-PG qui ont suivi des essais, en particulier T. Simmoneau et J. De Battista.

\section{RÉFÉRENCES}

Abbe E.C. \& Phinney B.O. (1951) The growth of the shoot apex in maize : external features. Am. J. Bot. 38 , 737-744

Blackman P.G. \& Davies W.J. (1985) Root to shoot communication in maize plants of the effects of soil drying. J. exp. Bot. 36, 39-48

Boiffin J., Sebillotte M. \& Couvreur F. (1976) Incidence de la simplification du travail du sol sur l'élaboration des rendements du blé et du maïs. Colloque ITCF : "simplification du travail du sol en production céréalière», 239-280

Bonhomme R., Derieux M., Duburcq J.-B. \& Ruget F. (1984) Variations in ovule number at silking in various corn genotypes. Maydica XXIX, 101-107

Bonnet O.T. (1940) Development of the staminate and pistillate inflorescences of sweet corn. J. Agric. Res. 60, 25-37

Derieux M., Bonhomme R., Ruget F. \& Duburcq J.-B. (1984) Influence du génotype et du milieu sur le nombre d'ovules présents à la floraison. In : Physiologie du maïs. INRA Paris, 1984

Duburcq J.-B., Bonhomme R. \& Derieux M. (1983) Durée des phases végétative et reproductrice chez le maïs. Influence du génotype et du milieu. Agronomie 3, 941-946

Evans G.C. (1972) The quantitative analysis of plant growth. Studies in ecology, vol. 1. Blackwell scientific publications, Oxford
Gay J.-P. \& Ménétrier M.A. (1978) Morphogénèse des organes reproducteurs chez le maïs : échelle de notation des stades. AGPM-ITCF, Paris, $38 \mathrm{p}$.

Gay J.-P. \& Roger J.-P. (1982) Maïs : il faut préserver le potentiel de départ. Perspect. Agric. 63, 19-26

Heller R. (1978) Abrégé de physiologie végétale, tome II. Masson, Paris, 214 p.

Hernandez A. (1987) Compte rendu des essais croissance et développement du maïs. AGPM, Paris, 103 p.

Hunter R.B. (1980) Increased leaf area (source) and yield of maize in short season areas. Crop Sci. 20, 571-574

Juguet M., Derieux M. \& Duburcq J.-B. (1986) Variations observées dans l'état de développement des systèmes foliaire et racinaire chez la semence mûre de maïs. Agronomie 6, 299-304

Lachno D.R., Harrison-Murray R.S. \& Audus L.J. (1982) The effects of mechanical impedance to growth on the levels of ABA and IAA in root tips of Zea mays L. J. exp. Bot. 33, 943-951

Laubengayer R.A. (1949) The vascular anatomy of the eight-rowed ear and tassel of golden bantam sweet corn. Am. J. Bot. 36, 236-344

Lesaint C. \& Coïc Y. (1983) Cultures hydroponiques. 1 vol. La maison rustique, Paris, $119 \mathrm{p}$.

Navarro H. (1984) L'analyse des composantes du rendement du maîs. Application à l'étude de la variabilité du rendement dans une petite région. Thèse de Docteur-Ingénieur, INA-PG, Paris, 238 p + annexes Ragland J.L., Hattield A.L. \& Benoit G.R. (1966) Photoperiod effects on the ear components of corn, Zea mays L. Agron. J. 58, 455-456

Ruget F. \& Duburcq J.-B. (1983) Développement reproducteur des bourgeons axillaires chez le maïs : stades de différenciation, nombre de fleurs. Agronomie 3, 797-808

Schreiber H.A., Stanberry C.O. \& Tucker H. (1962) Irrigation and nitrogen effects on sweet corn row numbers at various growth stages. Science 135, 1135-1136 Skene K.G.M. \& Kerridge G.H. (1967) Effect of root temperature on cytokinin activity in root exudate of Vitis vinifera L.. Plant Physiol. 42, 1131-1139

Tanaka A. \& Yamaguchi J. (1972) Dry matter production, yield components and grain yield of the maize plant. J. Fac. Agric. Hokkaido Univ. 57, 71-132

Tardieu F. (1987) Etat structural, enracinement et alimentation hydrique du maïs. III - Disponibilité des réserves en eau du sol. Agronomie 7, 279-288

Tardieu F. \& Manichon H. (1987) Etat structural, enracinement et alimentation hydrique du maïs. I - Modélisation d'états structuraux types de la couche labourée. Agronomie 7, 123-131

Tollenaar M. \& Daynard T.B. (1978) Kernel growth and development at two positions on the ear of Maize (Zea mays). Can. J. Plant Sci. 58, 189-197

Tollenaar M. \& Hunter R.B. (1983) A photoperiod and temperature sensitive period for leaf number of maize. Crop. Sci. 23, 457-460

Weatherwax P. (1955) Structure and development of reproductive organs. In : Corn and corn improvement (G.F. Sprague ed.) Academic press inc. New York

Yamaguchi J. (1974) Varietal traits limiting the grain yield of tropical maize. IV. Plant traits and productivity of tropical varieties. Soil Sci. Plant Nutr. 20, 287-304 